\title{
Ueber die Bestimmung der organischen Substanz und der Salpetersäure im Brunnenwasser etc.
}

Von

\section{E. Reichardt.}

Die Untersuchung des Wassers in seiner Bedeutung als Nahrungsmittel hat in den letzten Jahren sehr bedeutende Fortschritte gemacht. Nimmt man Analysen zur Hand, nur wenige Jahre früher ausgeführt, so entdeckt man sehr bald Mängel, welche nur durch neue Prüfungen gehoben werden können. Es versteht sich von selbst, dass ich hier nicht auf die einzelnen mineralischen Bestandtheile blicke, da es in den meisten Fällen wohl gleichgültig sein kann, ob Rubidium oder Caesium nachgewiesen wurde oder nicht, sondern auf die Ermittelung von 2 Bestandtheilen, welche jetzt zu den nothwendigsten und unentbehrlichsten für die Beurtheilung von Wasser gezählt werden müssen, - die Bestimmung der organischen Substanz und der Salpetersäure. Beide Substanzen wurden zwar schon früher ermittelt und gehören theilweise, z. B. bei den Berliner Brunnen, zu den längst bekannten Bestandtheilen, allein die Bedeutung und Wichtigkeit derselben ist erst in nenester Zeit, bei der Beurtheilung der Genusswasser, erkannt worden.

Hinsichtlich der organischen Substanz ist die Methode von $\mathrm{K}$ ub el *j sicher empfehlenswerth: Prüfung mit übermangansaurem Kali in schwefelsaurer kochender Flüssigkeit, wobei für einen Theil übermangansaures Kali - 5 Th. organischer Substanz in Rechnung kommen. Jeder weiss, dass diese Berechnung mit grossen Mängeln und gewisser Willkür behaftet ist, und wer scrupulös sein will, gibt nur den Verbrauch an Chamaeleon an; aber immerhin ist sie eine brauchbare, auf die Versuche von Kubel, Wood and Andere gestïtzte Grundlage: Ebenso sind die Einwirkungen des übermangans. Kalis auf andere Substanzen längst erwiesen und namentlich von $F r a n k l a n d$ festgestellt worden, aber immer wird bei Prüfung von Wasser der Verbrauch von übermangans. Kali substanz erweisen, welche leicht oxydirbar darin enthalten ist und deshalb dem Sauerstoff bedürtigen thierischen Organismus nachtheilig

*) Diese Zeitschrift 1867. Bd. 6. S. 252. 
entgegentritt. Handelt es sich um Untersuchungen von Brunnenwasser und dergl., so ist meistens der Ausdruck organische Substanz vollständig gerechtfertigt and besitzen wir wenigstens bis jetzt keine zweite, auch umständlichere Methode, welche uns genaueren oder geeigneteren Einblick in die Verhältnisse gewährte.

Der Einwand des Vorkommens der salpetrigen Säure, obgleich dieselbe in grösserer Menge noch nicht erwiesen ist, dürfte gerade bei dem Verfahren nach $\mathrm{Kubel}$ beseitigt sein, wie auch die Anmerkung in dieser Zeitschrift bei Mittheilung der Methode nach Kubel richtig bemerkt.

Fine qualitative Prüfung auf salpetersäure kann nach dem jetzt gewonnenen Einblick bei Untersuchungen von Wasser nicht mehr genügen, da die Salpetersäure allgemein, z. B. in den Quellen der Kalkformation, nach den Untersuchungen von Boussingault und Anderen, normal vorkommen soll, einigermaassen grössere Mengen áber die ùntrüglichsten Zeichen von Infiltration oxydirter Stoffe abgeben. Schon in der Fassung des Ausdruckes dürfte es klar gegeben sein, dass eben nur eine quantitative Bestimmung der Salpetersäure den genügenden Aufschluss gewähren kann.

Die in der neueren Zeit eingehender geprüften Methoden der Bestimmung der Salpetersäure gestatten eine nur wünschenswerthe Genauigkeit. Man benutzt entweder die Reduction von $-\mathrm{NO}^{5}$ zu $\mathrm{H}^{3} \mathrm{~N}$ und Ermittelung dieses letzteren, wobei ich die von Siebert modificirte $\mathrm{Me}-$ thode gebrauche, ${ }^{*}$ ) oder die Abscheidung von $\mathrm{NO}^{2}$ durch Eisenoxydullösung, welche bis jetzt ihre grösste Genauigkeit in der Titrirung der aus $\mathrm{NO}^{2}$ wieder erzeugten $\mathrm{NO}^{5}$, nach $\mathrm{Schloesing}$, **) besitzt.

Endlich schlägt Marx***) vor, die Salpetersäure direct mit Indigolösung zu titriren, indem man 50 C. C. Wasser mit der doppelten Menge reiner, concentrirter Schwefelsäure versetzt und unmittelbar die Indigolösung zufügt.

0. Reich $\dagger$ ), dem wir eine grosse Zahl Wasserprüfungen aus Berlin und Leipzig verdanken, gebraucht die Methode von Pelo uze und Fresenius, $\dagger_{\dagger}$ ) und ermittelt nach der Entfernung von $\mathrm{NO}^{2}$ das noch rückgebliebene Eisenoxydul. Gegen letztere Methode wendet Fresenius selbst ein (S. 425): „Bei Titrirung einer Salzsäure enthaltenden Eiseñ-

*) Fresenius, quantit. Analyse, 5. Auflage. 1863. S. 431.

**) Desgl. S. 427 .

***) Diese Zeitschrift Jahrg. VII, s. 412 u. f.

†) Die Salpetersäure im Brunnenwasser und ihr Verhältniss zur Cholera. Berlin 1869.

fi) Dessen quantit. Analyse, wie oben S. 423. 
oxydullösung mit Chamäleonlösung erhält man nur dann richtige Resultate, wenn bei der Feststellung des Wirkungswerthes und beim Gebrauche der Chamäleonlösung dieselben Verhältnisse in Betreff des Salzsäuregehaltes, des Verdünnungsgrades und der Temperatur obwalten ", und erklärt dann die leicht eintretende, weitergreifende Reaction. O. Reich (S. 26 seiner Schrift) bespricht die gleichfalls hier in Rechnung zu ziehende organische Substanz, welche bei der Titrirung mit Chamäleon unbedingt mit eingreift und hält den möglichen Fehler für sehr gering, so dass er pro Liter höchstens $0,0001-0,005 \mathrm{Grm}$. betragen könne; er ist aber keineswegs so nichtssagend, denn Mueller erklärt (0. Reich S. 65) einen Gehalt von 0,004 Grm. Salpetersäure im Liter schon als bedenklich, und nach meinen Untersuchungen von einem Quellwasser kann ich nur beistimmen. Die zugestandene Fehlergrenze überschreitet sonach sogàr die Grenze der Güte von Trinkwasser.

o. Reich findet in Berliner Wasserproben im Liter bis $0,675 \mathrm{Grm}$. $\mathrm{NO}^{5}$ (Gerichtsstrasse $42 ; \mathrm{S} .47$ ) und in 27 Wasserproben von Leipzig bis $0,346 \mathrm{Grm}$., Zahlen, welche uns in kleineren Städten Wohnenden und an frisches, reines Quellwasser Gewöhnten ganz exorbitant erscheinen müssen, und gab dies Veranlassung, einige Versuche $\mathrm{zu}$ wiederholen. Deshalb veranlasste ich die Zusendung einiger von 0 . Reich untersuchten Leipziger Brunnenwasser und erhielt folgende Resultate im. Liter nach Grammen:

\begin{tabular}{|c|c|c|c|c|c|c|c|}
\hline \multirow{3}{*}{ Name des Brunnens. } & \multirow{3}{*}{$\begin{array}{l}\text { Ab- } \\
\text { dampf- } \\
\text { ruick- } \\
\text { stand: }\end{array}$} & \multirow{3}{*}{$\begin{array}{c}\text { Glüh- } \\
\text { verlust : }\end{array}$} & \multirow{3}{*}{$\begin{array}{l}\text { Organ. } \\
\text { Subst. } \\
\text { durch } \\
\text { Cha- } \\
\text { mae- } \\
\text { leon: }\end{array}$} & \multicolumn{3}{|c|}{$\mathrm{NO}^{5}$} & \multirow{3}{*}{$\begin{array}{l}\mathrm{NO}^{5} \\
\text { Reich } \\
\text { fand : }\end{array}$} \\
\hline & & & & & Nach & & \\
\hline & & & & Siebert & $\begin{array}{l}\text { Schloe- } \\
\text { sing }\end{array}$ & Marx & \\
\hline Rossplatz & 0,980 & 0,230 & 0,092 & 0,1431 & 0,1468 & 0,254 & 0,347 \\
\hline Dorotheenstrasse. & $1, \mathbf{1 6 0}$ & 0,250 & 0,107 & 0,1488 & - & 0,1818 & 0,070 \\
\hline Gerberstrasse . . & 0,470 & 0,090 & 0,037 & - & 0,0236 & 一 & 0,255 \\
\hline Tauchaer Strasse. & - & - & - & 0,18225 & 0,1839 & - & 0,324 \\
\hline Bettelbrunnen . & - & - & - & - & 0,2362 & - & 0,331 \\
\hline Burgstrasse . . . & - & - & - & - & 0,0506 & -- & 0,212 \\
\hline Magdeburg. Bahnhof & - & - & - & - & 0,0132 & 一 & 0,065 \\
\hline Wasserleitung . . & - & - & - & $\ldots$ & 0,0115 & - & - \\
\hline
\end{tabular}

Das Wasser dèr neuen Wasserleitung zu Leipzig enthielt demnach 11,5 Th. Salpetersäure in 1 Million Theilen Wasser, während die Grenze von gutem Trinkwasser schon bei $4 \mathrm{Th}$. liegen soll! Gebirgswasser aus der Nähe von Liegnitz ergab mir nach derselben Methode gar keine Salpetersäure, das Wasser der Katzbach enthielt dort 5,06 Theile in 1 Mill. Theilen. 
Das Wasser der Tauchaer Strasse wtrde gleichzeitig durch Titriren des Eisenoxyduls bei der Salpetersäurebestimmung geprtift uud ergab darnach 0,5625 Grm. $\mathrm{NO}^{5}$, $\mathrm{Reich}$ fand 0,324 und nach Siebert und Schloesing wurden 0,18225 und $0,1839 \mathrm{Grm}$. erhalten. Eine Uebereinstimmung mit den Resultaten $\mathrm{R} e \mathrm{i} \mathrm{ch}$ 's kann natürlich nicht verlangt werden, da die Salpetersäure liefernden Einflüsse gewiss sehr wechseln; meine Wasserproben waren im Februar d. J. entnommen.

Um die Brauchbarkeit einer Methode für gegebene Zwecke zu controliren, genügt niemals eine Wiederholung der Versuche mit einem salpetersauren Salze, da sich bei diesem natürlich die günstigsten Verhältnisse bieten müssen. Die Controlversuche nach den Methoden von Siebert, Schloesing, Pelouze und Fresenius ergeben gleich genaue Resnltate und müssen sie bei genauer Beachtung und Betrachtung ergeben; anders verhält es sich bei Gemischen, bei denen mannigfache und gerechte Einwürfe gemacht werden können. Die Methode von Schloesing hat sicher das für sich, dass das erhaltene $\mathrm{NO}^{2}$ eben nur von $\mathrm{NO}^{5}$ oder $\mathrm{NO}^{3}$ herrühren kann und bei einiger Uebung geht die Prüfung rasch und äusserst sicher. Beiläufig will ich bemerken, dass an Stelle der ausgezogenen Kochflaschen, mit ganz gleichem Erfolge, gewöhnliche Flaschen mit Kautschukkork und Glasrohr genommen werden können.

Es war nicht meine Absicht, die Untersuchung der Wasserproben nach sämmtlichen Methoden vollständig auszuführen, die Differenzen bei der Prüfung nach Marx waren so bedentend, dass ich dieselbe bald aufgab, der Unterschied bei der Bestimmung des Eisenoxyduls desgleichen, dagegen zeigen die 2 Bestimmungen nach Siebert and Schloesing eine nur wünschenswerthe Uebereinstimmung, wie nicht anders $\mathrm{zu}$ erwarten.

Es wurden hierbei stets 500 C.C. Wasser mit kohlensaurem Natron bis zum Vorwalten versetzt, zur Trockene verdunstet und dann der Trockenrückstand auf Salpetersäure untersucht. Das Verdunsten mit Natron ist bei der Methode nach Siebert namentlich nothwendig, um vorher vorhandenes Ammoniak zu entfernen. Gegen die Titrirung mit Indigolösung nach Marx ist noch einzuwenden, dass bei kleinen Mengen NO ${ }^{5} 50$. C.C. Wasser zu wenig sein würden, bei Uebertragung anf 1 Mill. Theile wenigstens sehr erhebliche Unrichtigkeiten entstehen dürften. Vorläufig halte ich die Methoden nach Schloesing und Siebert als die geeignetsten und sichersten für die Bestimmung der Salpetersäure im Genuss- oder Betriebswasser. 
Die hier aufgeführten Bestimmungen der Leipziger Brunnenwasser wurden von meinen beiden Assistenten, Dreykorn und Scheermesser, bewerkstelligt.

\section{Modification des Piknometers.}

\section{Von}

\section{Dr. Wilh. Friedr. Gintl.}

Docenten für Chemie an der k. k. Universität zu Prag.

Jeder der irgend öfter in die Lage gekommen ist, das specif. Gewicht fester oder flüssiger Körper mit Hilfe des Piknometers bestimmen zu müssen, wird mir ztustimmen, wenn ich behaupte, dass dergleichen Bestimmungen immer ihr Missliches haben und ich dürfte wohl kaum der Einzige sein, der gerade dieser Methode der Bestimmung specif. Gewichte den Vorwurf einer zu dem Grade der erreichbaren Genauigkeit in keinem Verhältnisse stehenden Umständlichkeit macht. Von den gebräuchlichsten Formen des Piknometers sind jene, welche zur Erreichung einer vollständigen Füllung mit einem längeren oder kürzeren, durchbohrten Glaspfropfen, so wie die, welche mit einer durchbohrten Platte verschliessbar sind, allerdings, namentlich da, wo nur wenig Substanz zur Verfügung steht, oder es sich um flüchtige Substanzen handelt, jenen Formen vorzuziehen, wo die Grösse des Volums durch Auffüllen bis zu einer Marke oder Ablesen an einer Skala bestimmt wird; indess haften denselben immerhin Uebelstände an, die leicht $z u$ irrigen Resultaten führen können. So ist es beispielsweise kaum möglich, zumal bei Anwendung einer etwa leichter flüchtigen Flüssigkeit, in Folge der fortwäbrend statthabenden Verdunstung des Piknometerinhaltes, ein constantes Gewicht zu bekommen, und wenn schon dieses Moment die. Sicherheit der Gewichtsbestimmung illusorisch macht, so ist das um vieles mehr bezüglich des Umstandes der Fall, dass sich bei dem vor der Wägung nöthigen sorgfältigen Reinigen des Piknometers an der Aussenseite u. s. f. ein Anfassen desselben und also eine durch die Körperwärme bedingte Temperaturerhöhung des Piknometers und seines. Inhaltes kaum vermeiden lässt, deren nächste Folge die sein wird, dass ein Theil der durch die Wärmezufuhr ihr Volum vergrössernden Flüssigkeit aus dem Piknometer austreten wird, und dieses also nach dem Wiedereintritt der vorigen Temperatur in Folge der nunmehr eintretenden Volumenverminderung der Flüssigkeit von dieser nicht mehr völlig erfüllt werden wird. Deshalb ist es ja anch eine der Hauptregein für derartige Bestimmungen specif. Gewichte, sei es flüssiger Körper, sei es fester, das Piknometer 